CASE

oF

\title{
ULCERATION OF THE PULMONARY ARTERY
}

INTO AN ABSCESS OF THE LUNGS.

WITH REMARKS BY JOHN DALRYMPLE, EsQ.

By William CROWfoOT, Jun., Esq., Beccles.

Communicated by JOHN DALRYMPLE, Esq., SURGEON TO THE LONDON OPHTHALMIC HOSPITAL.

READ APRIL $11 \mathrm{TH}, 1843$.

Mr. L. B., aged 36, of short stature, strumous diathesis, and the member of a large family, several of whom have died of pulmonary consumption, had from childhood suffered from difficult breathing, the consequence of repeated attacks of inflammation of the respiratory organs, to which he had always been peculiarly subject. In the autumn and winter of 1841 he suffered from cough with mucopurulent expectoration, frequently tinged with blood, 
and almost all the symptoms of phthisis; by rest, steadily-continued treatment, and a careful avoidance of exposure to cold, his more urgent symptoms were relieved ; and, being naturally energetic, he returned to his usual professional avocations, those of a medical man, in the spring and summer of 1842 ; but he still continued to cough frequently, and expectorated every morning a considerable quantity of muco-purulent matter, occasionally tinged with blood; in this state he continued till the end of November 1842 ; when, after some considerable excitement, he was attacked with active hæmorrhage, which recurred almost daily, and sometimes in very considerable quantities, varying from half a pound to two pounds. The blood was always expectorated without effort, and appeared almost to flow of itself into the mouth, producing a slight tickling sensation in the trachea, for the relief of which he made a little cough, which was always followed by the expectoration of more or less blood mixed with a little mucus. The physical signs presented by the chest at this period were as follows:- the ribs on the left side were contracted and flattened when compared with those of the right side; the upper part of the left side of the chest was dull upon percussion, and the respiratory murmur was absent in that situation; the right side presented no abnormal sound. The pulse varied from 60 to 80 pulsations in the minute; the urine not high coloured; the tongue slightly coated, with a shining 
unhealthy appearance in the mucous membrane of the pharynx. I need hardly say that residing in the house of a most intelligent medical friend, no remedial means were left untried which either professional ability or the kindest friendship could suggest, but all proved unavailing; increased faintness supervened upon the increasing attacks of hæmorrhage, and at the end of a month he sunk from the exhaustion consequent upon the repeated bleedings.

Post mortem examination of the chest twenty-four hours after death.-Externally the left side presented the same flattened appearance observed during life. Upon opening the cavity of the thorax, numerous adhesions were found between the pleura pulmonalis and costalis of the left side, and the pleura investing both lungs had that peculiar spotted appearance arising from the deposit of strumous matter which is constantly observed in scrofulous subjects. The upper part of the left lung was entirely occupied by a large cavity containing about half a pound of grumous and coagulated blood; the walls of the cavity were composed of the parenchymatous structure of the lungs, condensed and solidified by pressure. Upon careful examination we found the pulmonary artery opening into the cavity at the distance of two inches from its bifurcation by an aperture as large as a crowquill. The right lung was healthy, with the exception of the strumous spots already alluded to. 
Some few points of interest not having been noted in the foregoing letter, Mr. Crowfoot subsequently added, "that the communications between the cyst and the bronchial tubes were numerous but not large. There was no disease or ulceration of the mucous lining of the trachea, which we, in common with all his medical friends, had been led to expect, owing to his constantly referring his pain and uneasy sensations to that organ."

Mr. Beales, of Halesworth in Suffolk, the kind and intelligent medical friend with whom L. B. resided, subjoined the following particulars :-

"The pulmonary artery was so large, as at first to be taken for the aorta, for it had a complete curvature to the right, as high up, and upon a line with the clavicle of the left side. The opening into the abscess, it is probable, was the result of the coats of the artery giving way, rather than the effect of the ulceration of the abscess ; as the opening from the artery was funnel-shaped, and terminated in the smallest possible aperture."

This case is submitted to the consideration of the Medico-Chirurgical Society, as one abundantly interesting in its details, and as affording an unequivocal instance of the communication of a large artery with the cavity of an abscess. The dilatation of the pulmonary artery may perhaps be attributable to pressure of the cyst upon one of its main divisions, and whether the communication between it and the abscess be due to the ulceration of its coats from pressure, or from bursting of its dilated and thin 
parietes, is a matter of far less moment than the establishment of the fact, that no vessel in the body is exempt from the consequences of pressure, and of the disintegrating influence of suppurating cavities in their immediate neighbourhood.

Abundant examples of the ulcerations of arteries of the systemic system have now been published, and Mr. Bloxam's paper shows that the large veins may be similarly circumstanced. This interesting case of Mr. Crowfoot completes the deficiency in the chain, by exhibiting a fatal hæmorrhage from the communication of a large branch of the pulmonary artery with a chronic abscess of the lungs. 